\title{
PROPOSALS OF EUROPEAN CITIZENS FOR REVIVING THE FUTURE OF SHRINKING AREAS
}

\author{
FlaVio Besana (10 1,2 \\ ${ }^{1}$ Spatial Foresight, Heisdorf, Luxembourg \\ ${ }^{2}$ Faculty of Human Geography and Planning, Adam Mickiewicz University, Poznań, Poland
}

Manuscript received: September 28, 2021

Revised version: October 13, 2021

\begin{abstract}
BesanA F., 2021. Proposals of European citizens for reviving the future of shrinking areas. Quaestiones Geographicae 40(4), Bogucki Wydawnictwo Naukowe, Poznań, pp. 15-28. 3 figs, 2 tables.

AвSTRACT: Shrinkage, depopulation and the related structural decline threaten development trajectories of more than a quarter of European territories from the present until 2050. In April 2021, the European Commission has launched the Conference on the Future of Europe to involve citizens and players beyond the traditional actors in shaping future policy agendas. The initiative consists of a wide-scale citizen engagement policy offering them a digital framework to actively contribute to the most relevant debates from April to December 2021. Given that shrinkage is a neglected theme in traditional policy arenas, this article examines the proposals of European citizens for reviving the future of shrinking areas. Through content analysis, the article highlights a limited relative presence of shrinkage in the Conference debate. Nevertheless, the results offer insights into the thematic concentration and the affinity of shrinkage with the most popular policy debates. The article also discusses the content of citizens' ideas for the future of shrinking areas, thus offering concrete proposals that may fuel the definition of future policy agendas.
\end{abstract}

KEYWORDS: shrinkage, depopulation, Conference on the Future of Europe, content analysis, citizen engagement, European policies, shrinking areas

Corresponding author: Flavio Besana, Spatial Foresight, 7, rue de Luxembourg, L-7330 Heisdorf, Luxembourg;

e-mail: flavio.besana@spatialforesight.eu

\section{Introduction}

Shrinking cities have been a growing topic of investigation for researchers and practitioners in the EU and beyond for the past two decades (Pallagst 2010; Rink et al. 2009; SCiRN 2008; Wiechmann 2009). The concept of shrinkage as a multifaceted process has been defined from different perspectives (Bontje, Musterd 2012; Haase et al. 2014; Wiechmann, Wolff 2013), and research agendas have been framed to deepen the knowledge around a broadening circumstance (Hollander et al. 2009). Shrinking areas are fragile places (Wolff et al. 2017), more vulnerable to challenges and therefore deserving special care during policy design phases (Großmann et al. 2013; Stryjakiewicz, Jaroszewska 2016). Shrinkage is not limited to an urban dimension, as in many cases the spiral of structural decline involves larger territories with different features (Copus et al. 2020; Hoekveld 2012), and research shows that rural areas are particularly affected in Europe (Copus et al. 2020; ESPON, 2017).

Despite the growing attention which has expanded the volume of research and its results, shrinkage has a hard time in climbing the priority 
list of policy makers at least at supra-local level (Bernt et al. 2014). Recent research confirms that shrinkage has been growing steadily in the past three decades and it will continue on this trend in the upcoming three decades until 2050, also enlarging to more EU territories than now (Besana, Böhme 2020). Notwithstanding its efforts, it seems that European policy-making has not yet answered the growing challenges of local population decline with a strategic focus and structured approach.

The recent developments in the context of the EU Cohesion policy seem to reflect a renewed attention to the phenomenon of depopulation. The Regulation of the European Regional Development Fund 2021-2027 includes for the first time a definition of what is a territory affected by a demographic decline: provinces or municipalities - NUTS 3 or LAUs - with $<12.5$ inhabitants $/ \mathrm{km}^{2}$ or that have suffered an interannual demographic decline of $>1 \%$ during 2007-2017 (European Parliament and Council, 2021). These are indicated to receive attention by the Member States for developing specific local action plans in the new programming period. Whilst this constitutes a significant novelty for shrinkage in the European debate, it is the result of a watered-down compromise, as negotiations narratives suggest that the ambitious goal of $5 \%$ earmarked funds had to be scrapped from the Regulation in favour of the present non-binding formulation (Pazos-Vidal 2021).

Moreover, it has been immediately pointed out that inconsistencies and dispersion between concurring policies and lack of binding elements within them signal an inbuilt resistance to recognise these areas facing structural decline. The limited political interest and the narrowness of the policy constellations behind this theme have limited the potential of a true change of pace for shrinkage in EU policy making (Pazos-Vidal 2021). Symptomatic of this is the lack of reference to the definition in relevant policy documents in the field (European Commission, 2021). Despite a partial deception and several doubts about implementation and impact, a small milestone has been set with the inclusion of a definition of shrinkage in official regulations.

These recent developments offer a potential window in the policy debate to fuel the recognition of shrinkage and depopulation. Literature shows that in such a particular territorial setting, and in the current political era, citizens' opinions and proposals become extremely relevant (Rodríguez-Pose 2018). A timely opportunity to support and valorise their contribution is the Conference on the Future of Europe recently launched (19th April 2021), which enriches the debate with a local perspective and has the objective to translate citizen proposals into policy priorities.

The Conference on the Future of Europe offers citizens a new public space for an open, inclusive and transparent debate about the future of actions and policies in the EU. Over the nine months of the Conference (April 2021-December 2021), citizens are called to directly intervene in setting the priorities of the policy agenda and traditional policy actors have the responsibility to synthesise the agenda and translate it into concrete measures and policy instruments (European Commission, 2020b). The Conference of the Future of Europe can be described as a process of agenda-setting carried out by unconventional policy actors (Fischer, Miller 2017). It represents an unusual policy initiative offering an EU-wide exercise of citizen engagement that allows for cross-border exchange of ideas and interactions among citizens.

The Conference on the Future of Europe is an ongoing exercise. The platform has been launched on April 19, 2021 and it will be closed on the 31st of December 2021. It identifies the following macro-themes for which citizens are called to enrich the policy debate through their ideas: Climate change and the environment; Health; A stronger economy, social justice and jobs; EU in the world; Values and rights, rule of law, security; Digital transformation; European democracy; Migration; Education, culture, youth and sport.

This article aims at providing insights for the following questions: are EU citizens concerned about the depopulation and the downward spiral of many territories when thinking about the future of the EU? Is the challenge of shrinkage perceived by citizens and which trends and themes is it mostly associated with? What ideas do citizens propose to mitigate this ongoing phenomenon? At the same time, the study also 
allows reflecting on the perception of shrinkage and depopulation outside academia and the policy debate.

The introduction has set the context and the aim of the research. The rest of the article is organised in three main sections. Section 2 outlines the data collection process, the choice of content analysis as the core methodology, including the illustration of the coding protocol. Section 3 presents and discusses the results of the article. Three subsections differentiate the discussion of results between (I) the relevance of shrinkage and depopulation in the Conference; (II) the thematic concentration of ideas and languages of the debate; (III) the content of citizens' proposals to address shrinkage. Section 4 reflects on the results and concludes.

\section{Materials and methods}

This article analyses an EU's policy initiative of citizen engagement, focusing on the so-called ideas proposed by citizens in the Conference on the Future of Europe. The purpose of the study is to research the presence of shrinkage or depopulation as a direct, or indirect topic in the debate. The research interest lies in understanding whether EU citizens have been reflecting about depopulation or shrinkage when shaping their ideas about the future of Europe. This analysis considers the first period of activity of this wide citizen engagement tool: 19th April 2021-15th June 2021. The preliminary assessment of an already very consistent number of ideas would allow publishing the results whilst the platform is still open, thereby favouring the emersion of neglected topics.

To provide an answer to the research questions, qualitative content analysis is identified as the most appropriate methodology (Flick 2014; Krippendorff 2018; Prior 2014). Nonetheless, the type of data and the research questions require that the analysis and the findings present also semi-quantitative elements in terms of occurrence and frequencies of concepts and words (Schreier 2014). Similarly to existing studies concerning content analysis in the field of EU policy, whilst the approach is essentially qualitative, the analysis cannot prescind from involving quantitative steps (Hecker et al. 2019; Venghaus, Hake 2018).

The methodology consists of the application of content analysis to all EU citizens' ideas posted on the digital platform of the Conference on the Future of Europe between the launch date 19th April and the 15th of June 2021. Following Scheirer (2014), the qualitative content analysis follows an open coding approach since the pilot phase. Then, the coding protocol has been evaluated and modified in the second round of coding, including the specification of sub-categories (Gibbs 2014; Venghaus, Hake 2018). The coding protocol has been refined in the analysis phase (third round) to better fit the data and its suitability to answer the research question. A fourth and last round of coding has sharpened the coding protocol to improve the presentation of findings (Schreier 2014).

The citizens' ideas in the Conference on the Future of Europe constitute an unconventional type of data in the context of policy content analysis. The format, the length and the tone of the data collected resemble closely that of Twitter and Facebook posts. Thus, from a technical standpoint, we are dealing with a content analysis of social media material, which is another prominent field of application for content analysis literature (Snelson 2016).

However, differently from social media platforms, the Conference on the Future of Europe has a declared purpose to shape the future policy agendas, and contributions are expected for a set of pre-defined themes. The audience is also slightly different: whilst for social media, the public is not predefined and, in most cases, restricted to existing connections, the Conference is visible to an unrestricted public that can also interact. At the same time, EU policy makers represent the main declared targeted audience (European Commission, 2020b).

Methods of content analysis in policy sciences have been applied to mass media and news reports material (Howland et al. 2006; Sarainsky 2015). However, whilst there is literature on the role of social media in the political debate, content analysis is scarcely applied to investigate the interplay between social media types of data with policy processes. This paper proposes to apply a methodology that is widely used for social 
media (Snelson 2016) to a different media context and in an experimental phase of agenda-setting within EU policy cycles. Besides the specific research interest on shrinkage and depopulation, elements of novelty stem from the unusual type of actors, data and format of the policy process under investigation.

To analyse the data, the software MAXQDA is used. The software allows for coding with a good degree of flexibility along the research process. It favours the adaptation of the coding protocol as the subsequent rounds of analysis contribute to refine or redefine the analytical framework. This is especially important when an inductive approach and open coding are chosen.

This section continues displaying how the data collection has been conducted; then it illustrates the final coding protocol with more detail so as to explain the rationale adopted for the content analysis of this policy initiative.

\section{Data collection}

The Conference on the Future of Europe (digital platform) is the unique data source for this study. The data collection is performed thanks to an automated computing process from the 'ideas' section of the website (https://futureu.europa. $\mathrm{eu} /$ ). The automated data collection process relies on python scripts to transfer the data (citizen ideas) from the website to pre-categorised word documents.

In the period 19th April 2021-15th June 2021, a total number of 4,576 ideas have been collected for the policy content analysis. The ideas are collected in ten separate documents, each corresponding to one of the main themes identified by the EU Commission, the EU parliament and the Council (European Commission, 2020b). The coding is performed in the original language for English, French, Spanish and Italian. For all the remaining languages the coding is performed on the English translation of ideas, carried out through Microsoft Word translator.

While the period of analysis does not cover the full length of the Conference on the Future of Europe, a sharp reduction has been observed in the pace of publications of new ideas in the weeks following our cut-off date (15th June) concerning the first eight weeks. The following eight weeks (until September 5) have increased the ideas by
2,479 , roughly half the amount of the initial eight weeks. At the same time carrying out the analysis of an ongoing policy debate also stands for an opportunity to continue or start contributing significantly before its end. When the results are disseminated rapidly, more ideas can be raised around the topic of shrinkage, which will result in greater attention from the EU institutions when concluding the Conference.

\section{The coding protocol}

To answer the research question, the ideas are analysed with an open coding approach. The choice of an open coding approach under the guiding question has to do with the unconventional type of data. In fact, ideas on the platform present important differences in length, language and register that in most cases do not resemble the existing academic and practice literature on shrinkage. Four rounds of coding are performed to refine or redefine the codes identified. In this way, the codes have been better suited, and the groups sorted more precisely so as to reflect the relevance of shrinkage in the Conference debate.

The first distinction is drawn at the surface between three main families of ideas and their relation to shrinkage or depopulation:

- Supporting ideas: ideas that directly or indirectly propose potential solutions to address shrinkage or depopulation.

- Contrasting ideas: ideas that would be harmful to shrinkage and depopulation typical issues, or ideas that advocate for depopulation as a positive trend to be further accelerated.

- Neutral ideas: ideas with no direct, nor indirect connection with the issues of shrinkage and depopulation and ideas with a too broad or generalised scope of applicability.

Supporting ideas reveal the relatedness of shrinkage with respect to other topics and priorities. Being the most relevant but complex code to assess, it is unpacked into three different subcodes, each with additional sub-codes. Such a branch system is designed to better streamline the complexity of shrinkage and embrace as many topics that could potentially have a positive impact on shrinkage dynamics. The coding of supporting ideas branches as follows: (I) direct support for shrinkage, depopulation, or ageing; (II) indirect support or close affinity with 
shrinkage; (III) support for remote and sparsely populated territories.

Shrinkage, depopulation, or ageing: The first branch offers the most direct connection to the research question as it consists of those ideas that directly address the topic of shrinkage, depopulation, or ageing. The ideas collected under this code specifically debate the problem of demography in the EU in a large sense or referring to a specific territory. The key distinction between the present and the rest of the codes lies in the $d i-$ rect reference to either shrinkage, depopulation or ageing in the text. Amongst these ideas, two subcodes will be distinguished in the result section: (I) awareness; (II) proposals.

Indirect support or affinity with shrinkage: the second branch collects those contributions that either propose ideas of general character with a clear territorial perspective in favour of disadvantaged territories or those ideas that directly discuss the issue of lagging regions and structural gaps. It is important to note that, despite the thinking behind these proposals might collide with shrinkage typical issues, they do not include any direct reference to demography (shrinkage, depopulation, or ageing). This branch will be further specified in the result section by the sub-codes: (I) digital relocation of labour; (II) structurally weak regions; (III) re-utilisation of infrastructure.

Support for remote and sparsely populated territories: the third branch recollects ideas in support of rural and remote areas typical issues, or ideas advocating for a re-localisation of life choices and the revamping of local economic activities at the detriment of multinational trade schemes. Despite the affinity of shrinkage with remote and rural contexts, no strict reference to demography is recorded in these branch ideas. Nonetheless, the potential relevance of the coded ideas and the affinity with shrinkage can be drawn from literature (Copus et al. 2020). This branch will be further specified in the Results section by the sub-codes: (I) remoteness and ruralism; (II) re-localism and deglomeration

Contrasting and neutral ideas codes contributions that are either neutral or have a direct and explicit negative impact on the shrinkage spiral. For the latter, only ideas with a strict explicit connection with shrinkage have been here considered. In the Results section, the code is further specified.

\section{Results and discussion}

The analysis of the policy content reveals that the presence of shrinkage and, more generally, of depopulation and lagging territories seems limited in the contributions from EU citizens in the Conference on the Future of Europe.

This section addresses first the significance of shrinkage-related ideas and the results of the coding iterations, then, in the second sub-section, the thematic concentration and language of the proposals discussed. In the third sub-section, the content of the most relevant ideas for shrinkage is illustrated through some examples.

\section{Shrinkage- and depopulation-related ideas in the Conference on the Future of Europe}

This section discusses first the incidence and the relevance of shrinkage-related ideas in the Conference for the Future of Europe. Then, it illustrates the distribution of such shrinkage-related ideas across the different sub-codes of the protocol.

The total number of ideas coded amounts to 4,576 across the ten different themes categorised by the EU Commission in the digital platform in the period under scrutiny (19th April 2021-15th June 2021). On the total number, only 119 put forward ideas that support directly, or indirectly the issue of shrinkage. Figure 1 illustrates the relatively low attention arising from shrinkage-related issues in the Conference on the Future of Europe.

Figure 1 shows that most ideas for the future of the EU do not have any direct, or indirect relation to shrinkage typical issues. Such low incidence $(2.6 \%)$ becomes particularly interesting when considering the number of people that already today live in declining urban and rural contexts.

It can be estimated that at least $19.4 \%$ of the European population lives in a shrinking municipality. The estimation is definitely conservative as, following one of the most widely used shrinking city definitions (SCiRN 2008; Wolff, Wiechmann 2018), it takes into account only municipalities that are $>5,000$ inhabitants. It means that the percentage of people living in shrinking contexts would raise when considering smaller municipalities, rural and sparsely populated 


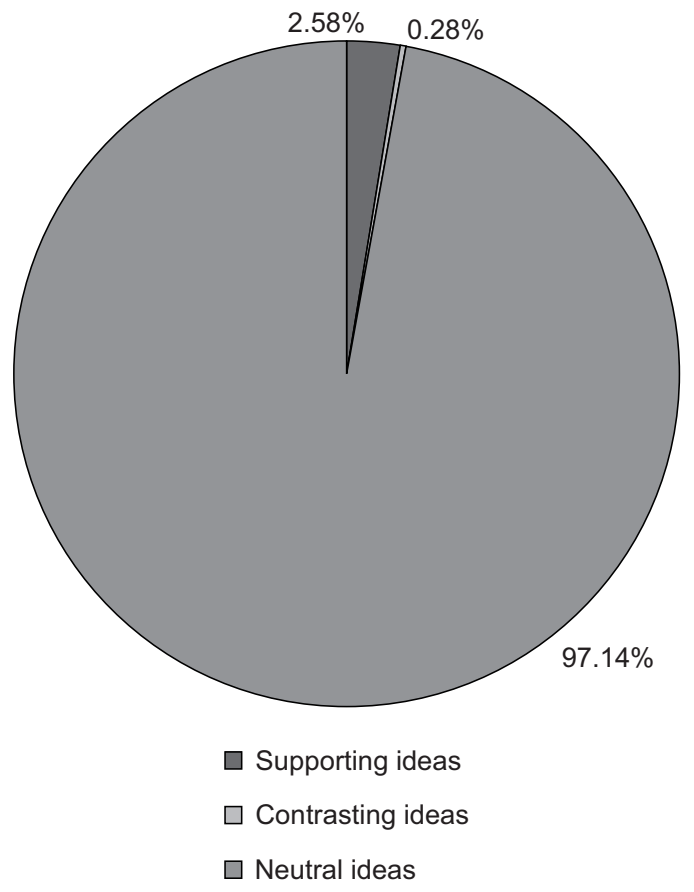

Fig. 1. Relevance of shrinkage in citizens' ideas in the debate on the future of Europe.

Source: own elaboration based on data from the Conference on the Future of Europe.

areas, that are often associated with shrinkage episodes (Copus et al. 2020; ESPON, 2017).

The pure numbers are, of course, not easily comparable as we are talking about ideas on one side and population on the other. Nevertheless, a limited incidence of shrinkage-related ideas alone and the volume of people living in such contexts seem worth highlighting in shrinking cities and regional studies debates.

The reasons why shrinkage-related problems do not attract greater attention from citizens can be various and opposing. Taking the most recent literature into account, it can be inferred that it has to do with the feeling of being left behind, which seems to drive citizens away from a European future towards a future of nations (Rodríguez-Pose 2018, 2020). In such interpretation the habitants of shrinking areas lean more towards nationalist sentiments which are often associated with Euro-scepticism, thereby showing lower levels of participation in the debates on the future of the EU.

A different reading would suggest that the lack of contributions derives from a reduced engagement caused by a perduring situation of decline or by the lack of engagement structures and habits, which is totally unrelated to political beliefs. In this interpretation, citizens would feel like having less care about the future of their territories, or simply lack the initiative or the framework for giving their contribution (Hospers 2013).

Alternatively, it is possible that most citizens feel at ease with the shrinking situation and are simply not interested in changing the status. Whilst it is hard to find literature pointing in this direction, this option should not be excluded.

Different reasons seem plausible to explain the low involvement of European citizens with shrinking areas' futures. However, it is beyond the scope (and the methodology) of this study to retrieve the motivations underlying this apparent lack of interest. What is relevant for this article is to highlight that shrinking cities, despite being an issue of growing concern from past to future (Besana, Böhme 2020), do not attract citizens' attention as data and literature would have suggested.

Table 1 illustrates in more detail the distribution of shrinkage-related ideas across the different sub-codes identified. This layer of analysis will allow for a more detailed perception of citizens' ideas within the Conference on the Future of Europe.

Only 48 of the 119 coded ideas directly address the problem of shrinkage, depopulation, or ageing. Among these, results from the open coding approach suggest the following distinction:

- Awareness: A total of 20 ideas that discuss the demographic issue with the main objective of

Table 1. Coding results of Citizens' ideas in the Conference on the Future of Europe.

\begin{tabular}{|l|c|c|}
\hline \multicolumn{1}{|c|}{ Subsections of main code } & $\begin{array}{c}\text { Main } \\
\text { code }\end{array}$ & $\begin{array}{c}\text { No. of } \\
\text { ideas }\end{array}$ \\
\hline $\begin{array}{l}\text { Awareness-raising on shrinkage or } \\
\text { depopulation }\end{array}$ & 20 \\
\hline $\begin{array}{l}\text { Proposals to mitigate shrinkage or } \\
\text { depopulation }\end{array}$ & 28 \\
\hline Ruralism and remoteness proposals & 20 \\
\hline $\begin{array}{l}\text { Re-localism and deglomeration propos- } \\
\text { als }\end{array}$ & 26 \\
\hline Re-utilisation of infrastructure proposals & 3 \\
\hline Digital relocation of labour proposals & 6 \\
\hline Proposals for structurally weak regions & 16 \\
\hline Calling for further depopulation & 5 \\
\hline Urbanisation and agglomeration & 8 \\
\hline
\end{tabular}

Source: own elaboration based on data from the Conference on the Future of Europe. 
raising awareness and calling for the intervention of relevant public bodies.

- Proposals: A total of 28 ideas that, going a step further, acknowledge the demographic problem and propose solutions to address it either locally or at a wider scale. The content is presented with greater detail later on.

Among the remaining shrinkage-related ideas, a majority of 46 are proposals in support of rural, remote and sparsely populated areas. Whilst the rural dimension of shrinkage is perceived with greater emphasis, its urban dimension shrinkage does not seem to be prioritised by EU citizens in the Conference. As a result of the open coding iterations, the following sub-categories are distinguished:

- Remoteness and ruralism: A total of 20 ideas propose or advocate for solutions to the typical problems of remote and rural shrinking areas such as access to essential services, school and education availability, social and economic opportunities, mobility and attractiveness (Barca et al. 2014).

- Re-localism and deglomeration: A total of 26 ideas promote the benefits of living, working and consuming in non-urban contexts or ideas calling for the need to decentralise life choices for socio-economic and environmental reasons.

The remaining shrinkage-related ideas display an affinity with shrinkage, especially addressing the issue of structural decline. Even though not mentioning directly the demographic dimension, proposals addressing structural territorial weaknesses are closely relevant and recall the socio-economic disparities that are typical of shrinking areas. As a result of the open coding iterations, the following sub-categories are distinguished:

- Digital relocation of labour: Six ideas advocate for the flexible re-location of digital jobs that directly embed a territorial perspective. Whilst digital relocation of labour is a trending topic across the Conference on the Future of Europe, the ideas in favour of teleworking per se have not been included in this code as it is not possible to derive information about the geographical location of such jobs, which can also happen in metropolitan areas and fast-growing cities. The key element considered is the co-occurrence of job re-locations with a territorial perspective in favour of remote, forgotten places to reduce disparities and re-balance development opportunities.

- Structurally weak regions: A total of 16 ideas call for the reduction of structural gaps between EU territories. They directly refer to the problem of lagging regions and either call for action or propose solutions to re-distribute development opportunities.

- Re-utilisation of infrastructure: Three ideas propose the revitalisation of abandoned industrial sites, buildings or infrastructure in general. Despite no direct reference to demography, this very specific category of ideas addresses one of the problems most often characterising shrinking contexts.

All the rest of the ideas analysed by the paper are either negatively related to shrinkage or are neutral. The following codes help to distinguish among them:

- Calling for further depopulation: Five ideas advocate the need for a continuous reduction of population in the EU to preserve the environment and to re-install a sustainable demographic balance among generations. These ideas promote a consistent reduction of demography to relieve the pressure on the primary source and land deprivation in the EU.

- Advocacy for urbanisation and agglomeration: Eight ideas promote further urbanisation and greater agglomeration towards capitals and major cities. These ideas dwell on the need to concentrate services and infrastructure to increase efficiency and improve accessibility of services and network effects.

- Neutral ideas: All remaining ideas have no direct relation to shrinkage, nor affinity with shrinkage topics. The code recollects all ideas of broad applicability (no significant relation to demography or shrinkage) or those specific proposals that neither express any territorial nor socio-demographic thinking.

\section{Thematic concentration of shrinkage-related ideas and languages of the debate}

The present section starts by illustrating the language of shrinkage-related ideas to verify the presence of patterns or exceptional concentrations of interest on shrinkage in specific national debates. Figures are displayed in Table 2. 
Table 2. Language of shrinkage-related ideas in the Conference on the Future of Europe.

\begin{tabular}{|c|c|c|c|c|c|}
\hline Languages/topics & $\begin{array}{l}\text { Shrinkage, depop- } \\
\text { ulation, ageing }\end{array}$ & $\begin{array}{l}\text { Remoteness and } \\
\text { re-localism }\end{array}$ & $\begin{array}{l}\text { Indirect affinity } \\
\text { with shrinkage }\end{array}$ & No. of ideas & $\begin{array}{l}\% \text { on total ideas } \\
\text { per language }\end{array}$ \\
\hline English & 24 & 8 & 11 & 43 & 2.6 \\
\hline French & 6 & 14 & 4 & 24 & 3.7 \\
\hline German & 4 & 6 & 4 & 14 & 1.3 \\
\hline Spanish & 5 & 7 & & 12 & 4.8 \\
\hline Italian & 2 & 4 & & 6 & 2.3 \\
\hline Slovakian & 2 & 2 & 2 & 6 & 11.8 \\
\hline Portuguese & & & 3 & 3 & 4.4 \\
\hline Hungarian & 1 & 2 & & 3 & 4.3 \\
\hline Finnish & & 1 & 1 & 2 & 5.0 \\
\hline Latvian & 1 & 1 & & 2 & 33.3 \\
\hline Romanian & 1 & & & 1 & 2.6 \\
\hline Dutch & & 1 & & 1 & 0.6 \\
\hline Swedish & & 1 & & 1 & 2.2 \\
\hline Polish & & 1 & & 1 & 1.9 \\
\hline
\end{tabular}

Source: own elaboration based on data from the Conference on the Future of Europe.

The most diffused language in the debate on the future of shrinking areas is English. Unsurprisingly, it is most often used to reach a larger audience but becomes less relevant to identify specific local debates around shrinkage. The remaining distribution of the original language of shrinkage-related ideas shows the higher attention given by French speakers (24 ideas), followed by a good representation from German (14 ideas) and Spanish (12 ideas) speakers; Italian speakers have also contributed, though to a minor extent (six ideas). The contribution of Slovakian speakers (six ideas) to the debate is impressive when considering the size of the Slovakian population compared to all the rest. The remaining ideas display smaller contributions from Portuguese, Hungarian (three ideas), Finnish and Latvian (two ideas), and Romanian, Dutch, Swedish and Polish (one idea).

It is particularly relevant to relate the amount of shrinkage ideas to the total of ideas submitted per language. The last column of Table 2 displays the results of this calculation and reveals some insights on the relevance of shrinkage across EU national debates. Limitedly to the information available and within the perimeter of the Conference on the Future of Europe, it is worth noting that Latvian and Slovakian debates are showing the highest engagement in the future of shrinking areas. Spanish, Portuguese and Hungarian speakers still display a significant engagement. The remaining languages display a comparatively low engagement, while the languages absent from the table did not display any shrinkage-related idea.

The section continues by illustrating the thematic concentration of shrinkage-related ideas among the policy topics set by the Conference on the Future of Europe. This information is relevant to see what trend topics the future of shrinking areas is associated with. What could be the main drivers of intervention for the future of shrinking areas? What are the policy themes citizens feel more closely related to shrinkage? Figure 2 reports the frequency of ideas in support of shrinkage across the ten themes available in the Conference.

The thematic concentration reveals a rather neat association of topics with shrinkage. Ideas in the fields of environmental and social justice represent roughly $60 \%$ of the total. Whilst for economic, employment and social justice it is an expected result considering the typical struggles of shrinking areas, it is striking to observe that Climate change and the environment are even more often associated with shrinkage spiral. On a first interpretation, it reads that the path to a greener future for the EU goes at hand with better future perspectives for shrinking areas.

Climate change and the environment is the most common theme associated with ideas on the future of shrinking cities. EU citizens have most often associated the revamping of declining urban and rural contexts at the detriment of metropolitan areas with having a positive rebalancing of land and resources exploitation. In general, 


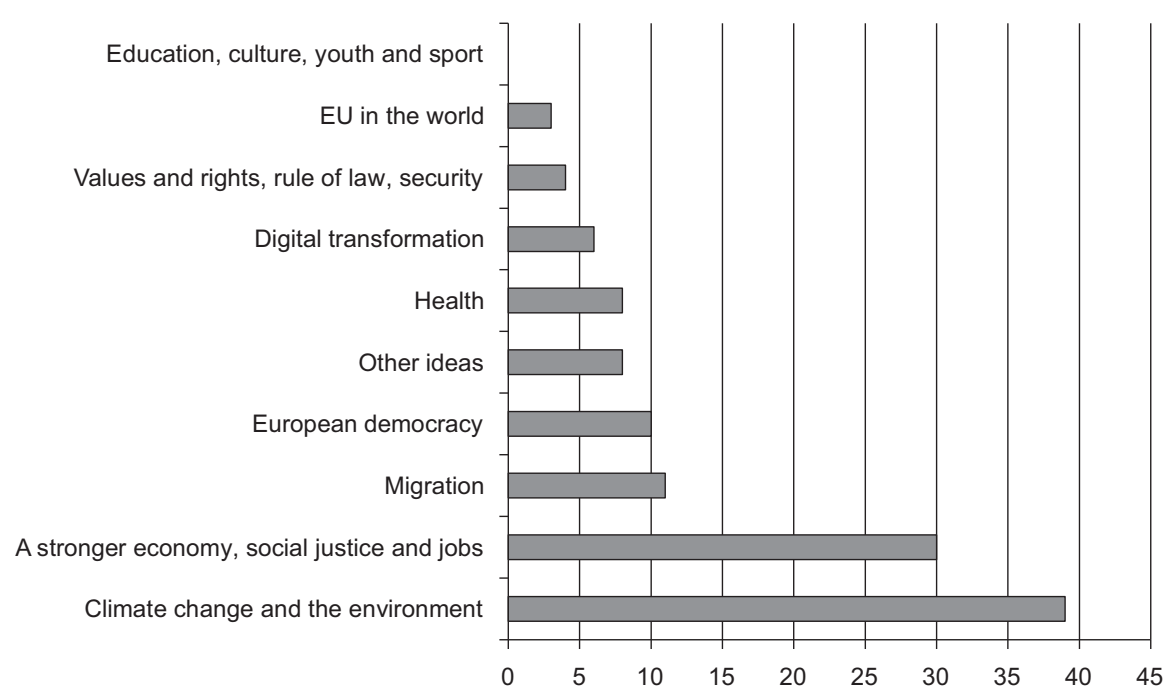

Fig. 2. Concentration of shrinkage-related ideas across themes of the Conference on the Future of Europe. Source: own elaboration based on data from the Conference on the Future of Europe.

citizens consider the objectives of climate mitigation actions compatible and benefitting the revival of declining areas, especially in rural domains.

A stronger economy, social justice and jobs come second for shrinkage-related ideas. Economic stagnation or decline, social inequalities and reduced job opportunities constitute the main features of shrinkage definitions in existing literature (Bontje, Musterd 2012; Hollander et al. 2009; Rink et al. 2009). The affinity of shrinkage with this theme is tight and lies at the origins of the phenomenon. Citizen proposals touch upon the territorial disparities suggesting greater investment in skills and innovation, as well as facilitated paths to create or access job opportunities.

Migration and emigration patterns at different levels are typically associated with shrinkage in both specialised and general literature (Haase et al. 2016; The Economist 2017; Wolff et al. 2017). Patterns of outmigration, both within countries and between EU countries are often cited among the major causes of shrinkage. In the Conference on the Future of Europe, this argument is strengthened but it is often enriched by an additional perspective on global migration schemes. Extra-EU immigration is proposed by citizens as a potential source of revitalisation for shrinking areas of the EU.

The idea in the remaining themes covers a wide spectrum of shrinkage-related problems with a more specific focus. Health and Values and rights, rule of law and security stress the shrinkage typical difficulties in accessibility to basic services, especially in rural and remote shrinking areas (Copus et al. 2020; ESPON, 2017; Verma, Taegen 2019). European democracy revamps the argumentation of forgotten and left behind places (Davoudi 2019; Rodríguez-Pose 2018) and the importance for them to have a bigger voice in the future of Europe. Digital transformation emphasises the structural gap in digital infrastructure that handicaps the development trajectories of shrinking areas (Barca et al. 2014; Copus et al. 2020; Haase 2012), especially in a changing world where remote working may constitute a major opportunity for their revitalisation.

In the next section, the article synthetises the most recurring ideas and concrete proposals that citizens have suggested to address the problem of shrinkage in the EU.

\section{Content of shrinkage-related proposals}

The results highlight a relatively limited relevance of shrinkage and shrinkage-related issues in citizens' contributions to the Conference on the Future of Europe. Then, the thematic concentration of ideas not only suggests traditional (service accessibility and industrial revitalisation), but also new perspectives (remote working and environmental) to think about the future of shrinking areas in the EU.

Notwithstanding the relatively scarce significance in overall volumes, the 48 shrinkage ideas, plus the additional 71 shrinkage-related ideas 
offer interesting material to reflect about the citizens' perception of the problem, and especially their proposed solutions. In a policy initiative that prioritises EU citizens' voices to shape the agenda of future policies and in general the future of Europe, their proposals gain extra emphasis and reach higher importance. In the following paragraphs, the content of citizens' proposals is presented.

The illustration of citizens' proposals prioritises contributions that have a direct target on shrinkage or structural disparities between regions, and actively proposes ideas that could revitalise such contexts. Following these criteria, a total of 51 ideas have been selected for their active contribution to shrinkage and depopulation debate within the Conference. The recurrence of very similar ideas enabled grouping them into representative categories. This last step, which can be methodologically described as a final round of coding applied on a restricted sample, makes it possible to synthesise the analysis and present the ideas in a coherent way. Figure 3 displays the frequency of citizens proposals grouped in the synthetic categories identified.

Results show that EU citizens, when contributing to shape the future of shrinking areas, prioritise the following ideas and opportunities for the revitalisation of such declining socio-economic contexts. Immigration and migrants' integration in the local reality is often (seven ideas) proposed as a unique opportunity to revitalise depopulating areas. Intra and extra EU immigrations are equally proposed, and a call for an orchestrated process, based on the labour and skills needed in each territory, is a common feature of ideas pointing in this direction.

With the same frequency (seven ideas), citizens' ideas suggest a Green Cohesion policy with depopulation criteria. These ideas call for specific employment incentives, subsidies, and support to promote a strategy of innovation and industrial revitalisation in depopulating territories. All these ideas stress the need to have a common denominator on sustainability that drives revitalisation strategies. Among this category, we find the proposal of a 'location-based quota' of funds with facilitated procedures that helps entrepreneurs and innovators to settle in shrinking areas. On similar wavelengths, we find ideas that propose compensations to rural and depopulating areas for their eco-system services and incentives for polluting companies to invest their due compensations in such areas.

Together with Immigration and a renewed cohesion spirit, citizens' proposals find most common ground on Remote working and tax adaptation for the service economy (seven ideas). The Covid-19 crisis has confirmed the possibility to delocalise a consistent number of tasks, and jobs in the service economy, thanks to remote working schemes. EU citizens identify this as a major opportunity to revitalise shrinking contexts, thanks to the relocation of workers towards less crowded, more natural, cheaper locations, but also the inversion of past migration paths from major cities to

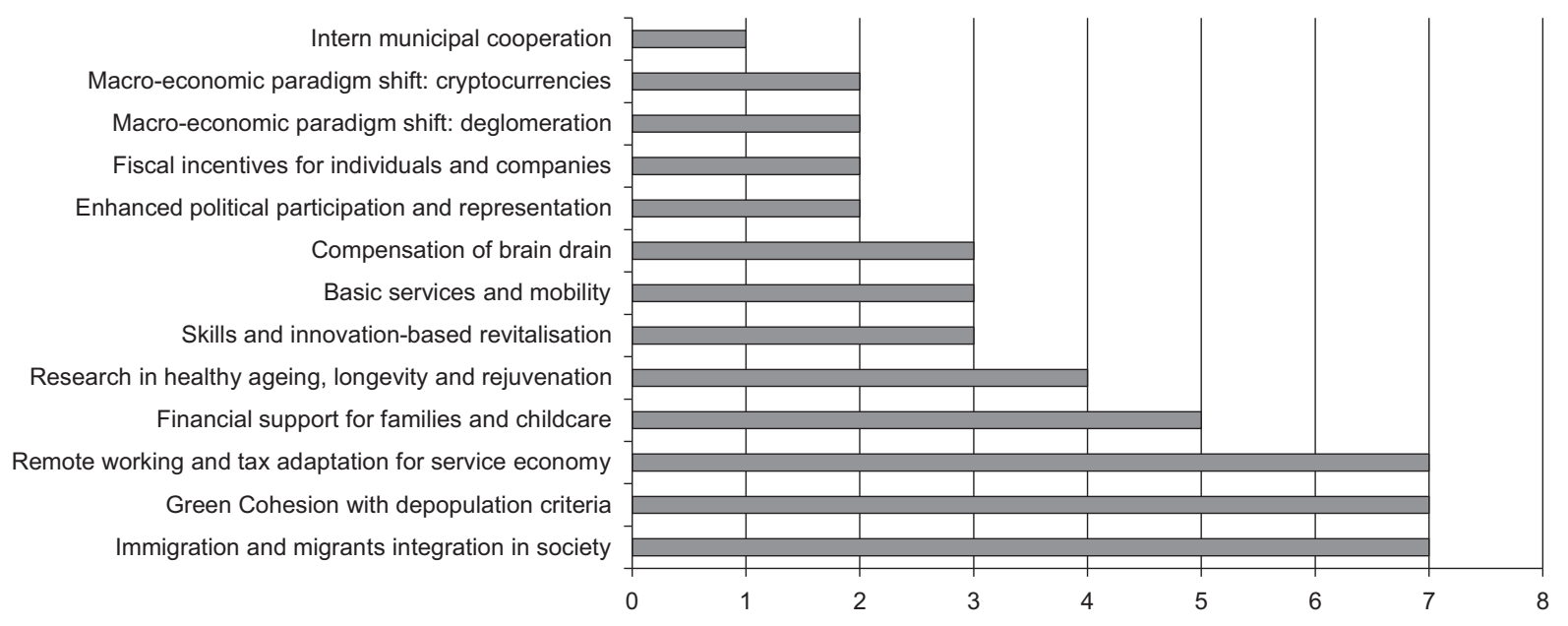

Fig. 3. Citizens' proposals to mitigate shrinkage and depopulation. Source: own elaboration based on data from the Conference on the Future of Europe. 
places of origin. These ideas converge also in the call for ad-hoc regulation from the EU to harmonise the possibility from a tax and social security perspective.

Financial support for families and childcare follows closely the previous ideas in terms of frequency among shrinkage-related ideas (five ideas). This category is centred on the decline of birth rates and its ideas advocate for greater financial (but also services) support for families to be able to raise more children. These ideas include not only direct help to families, and wider and more capillary access to childcare, but also incentive systems based on the number of children. For instance, ideas include a progressive scheme to anticipate retirement soils proportionally on the number of children. On the matter of childcare, ideas propose an active role of older generations in intergenerational education facilities hosting kids and retired people together. The relevance of older people in the debate is confirmed by the next category of ideas: research in healthy ageing, longevity and rejuvenation (four ideas). Differently from the previous category, the decline in birth rates is assumed irreversible, and proposals go in the direction of older people for revitalising shrinking areas. This category collects proposals advocating for greater investments in research for healthier ageing, longevity and rejuvenation therapies. A very elaborated proposal also suggests the foundation of a 'European Institute for Healthy Longevity Research'.

Some citizens' proposals highlight the need for greater public investment to fuel a skill and innovation-based revitalisation (three ideas) of shrinking areas, including re-skilling programmes, both practical and intellectual, for areas where traditional industries have been phased out but also direct subsidies and incentives for catalysing innovation. On the matter of knowledge skills, other proposals call for compensation schemes for brain drain (three ideas), both in the direction of so-called 'comeback' programmes for previously emigrated workers, but also in the direction of subsidies and direct investments in shrinking areas to attract new workers and incentive schemes to support their relocation. Improvement in the access to basic services and local mobility (three ideas) is also stressed as being the main critical factor in rural and remote shrinking areas. On these competencies, inter-municipal cooperation (one idea) is proposed as a possible solution, which calls for national or supra-national support in promoting such cooperation frameworks.

The remaining proposals to mitigate depopulation trends and revitalise shrinkage contexts include a greater Political commitment and representation (two ideas) for these territories, through regionalisation of political responsibilities and tighter links between national decisions and local representation; Fiscal incentives for individuals and companies (two ideas) to locate or re-locate in shrinking (or declining) areas; a shift in macro-economic paradigm is desired to revert agglomeration economies and privilege local and small-scale production and consumption chains (two ideas). On a similar note, Cryptocurrencies (two ideas) are proposed to be a powerful instrument against money concentration, able to re-distribute and re-equalise development opportunities across territories.

\section{Conclusions}

Shrinkage and depopulation have constituted an important challenge for territorial development in Europe (Gløersen et al. 2016; Hospers 2013; Wolff et al. 2017) and recent studies reveal that their impact is expected to grow further in the upcoming years (European Commission, 2020a; Directorate-General for Economic and Financial Affairs, 2020), with growing patterns of localised decline (Besana, Böhme 2020). Despite the growing attention which has expanded the volume of research and interest, shrinkage has struggled to climb the priority list of policy makers at supra-local level (Bernt et al. 2014). The latest developments in Cohesion policy would suggest that demographic decline has finally gained attention, but several doubts have immediately been cast on the inbuilt resistance to recognise the structural decline of shrinking areas, and the perduring lack of structured interventions (Pazos-Vidal 2021). In this policy context, the Conference on the Future of Europe constitutes an EU-wide initiative of citizen engagement, allowing them to influence the future policy agenda of the EU (European Commission, $2020 \mathrm{~b}$ ) and revamp the interest around socio-economic difficulties of shrinking areas. 
This article analysed citizen contributions in this debate through a qualitative content analysis of their ideas (Flick 2014; Prior 2014). Following an automated process of data collection, citizens' proposals have been assessed through a coding protocol, the result of an open coding approach in multiple iterations (Gibbs 2014; Schreier 2014). Results of the analysis display a limited relevance of shrinkage and depopulation in citizens' proposals for the future of Europe. Among the 4,576 contributions, only 48 directly address the demographic issue, plus 71 show affinity or indirectly support issues that are closely related to shrinkage. The relevance appears low when considering the extension of shrinkage phenomena, accounting for roughly $25 \%$ of EU territories and $20 \%$ of the EU population. Whilst the engagement of citizens in this debate is hard to interpret, possible concurring reasons for low participation on these issues can be traced to citizens' resignation and inability sentiment (Hospers 2013), or their abandonment feeling by institutions (RodríguezPose 2018, 2020).

Despite the limited relevance compared to the other themes, shrinkage and depopulation ideas constitute valid material to reflect on citizens' perception of the problem, and their proposals for the future of shrinking areas. The results of the thematic analysis suggest that the future of shrinkage has been most often associated with the following main policy challenges: (a) climate change, environment and sustainable resources exploitation; (b) economy, social justice and employment; (c) migration patterns, perceived both as cause and possible solution.

The article also analyses the content of citizen concrete proposals to revitalise the future of shrinking areas. These results may constitute a good base to fuel policy debates on shrinkage and help policy makers to shape their agendas when intervening in these fragile places. In synthesis, citizens see the greatest opportunities for revitalisation in: immigration and migrants' societal integration in local realities; in the fiscal adaption and institutionalisation of remote working schemes; in the financial support, incentives and childcare for families to raise more children; in the creation of re-skilling programmes driven by an innovation spirit that is both practical and knowledge-based; in compensation schemes and comeback programmes for brain drain as well as incentives for individuals and companies to relocate towards shrinking areas; in the research for healthier ageing and longevity. In addition, citizens ideas would suggest institutions to: adopt a new Green Cohesion spirit that includes location-based quotas with depopulation criteria; improve access to basic services, and local mobility, also through inter-municipal cooperation schemes; review their macro-economic paradigm in favour of deglomeration objectives and the adoption of cryptocurrencies.

This article sheds light on EU citizens' perception of shrinkage and their ideas for the future of these territories. In the meanwhile, the policy debate on these themes seems to receive increasing attention as the projections indicate that it will be a major challenge for Europe in the next 30 years. Considering the stakes, more research is needed to understand the citizens' perspective on this problem. This article only covers a tiny portion of the debate, monitoring needs to be continued within the Conference on the Future of Europe. More importantly, research in this direction needs to be extended beyond the boundaries of the Conference, as citizens are active locally or through different digital and non-digital frameworks.

\section{Acknowledgements}

I express my thanks for the financial support to RE-CITY ITN (Horizon 2020 - MSCA agreement No. 813803). I would also like to thank Kai Böhme and Spatial Foresight for the support in conceptualising the research, Norberto Soldano for the support in computing for data collection and the reviewers and the editors for their contributions.

\section{References}

Barca F., Casavola P., Lucatelli S., 2014. A strategy for inner areas in Italy: Definition, objectives, tools and governance. Materiali Uval Series. Online: https://www.agenziacoesione.gov.it/wp-content/uploads/2020/07/MUVAL_31_Aree_interne_ENG.pdf (accessed: 27 September 2021).

Bernt M., Haase A., Großmann K., Cocks M., Couch C., Cortese C., Krzysztofik R., 2014. How does(n't) urban shrinkage get onto the agenda? Experiences from Leipzig, Liverpool, Genoa and Bytom: Urban shrinkage on European policy agendas. International Journal of Urban and Regional Research 38(5): 1749-1766. DOI 10.1111/1468-2427.12101. 
Besana F., Böhme K., 2020. The future of shrinking cities in the EU: Pathways 1991-2050. ERSA 2020 conference: Spatial challenges for the new world, ERSA. Online: https:// ersa.eventsair.com/QuickEventWebsitePortal/ersawebconference2020/ersa2020/Agenda/AgendaItemDetail? id=811f8c45-0df1-9396-d292-39f0749ebabd (accessed: 27 September 2021).

Bontje M., Musterd S., 2012. Understanding shrinkage in European regions. Built Environment 38(2): 153-161. DOI 10.2148/benv.38.2.153

Copus A., Kahila P., Fritsch M., Dax T., Katalin K., Gergely T., 2020. ESPON ESCAPE (European Shrinking Rural Areas: Challenges, Actions and Perspectives for Territorial Governance), Final Report. ESPON. Online: https:/ / www. espon.eu/escape (accessed: 27 September 2021).

Davoudi S., 2019. From 'left behind' to 'kept behind' places: Re-imagining territorial cohesion (Keynote speech). ESPON Seminar on the role of functional areas for territorial cohesion, Iași, Romania. Online: https://www.youtube. $\mathrm{com} /$ watch?time_continue $=3799 \& \mathrm{v}=9$ XgHhgZbA34 (accessed: 27 September 2021).

ESPON, 2017. Shrinking rural regions in Europe: Towards smart and innovative approaches to regional development challenges in depopulating rural regions [Policy Brief]. Online: https://www.espon.eu/rural-shrinking (accessed: 27 September 2021).

European Commission, 2020a. Report from the Commission to the European Parliament, the Council, the European Economic and Social Committee and the Committee of the Regions on the impact of demographic change ( $\{\mathrm{SWD}(2020) 109$ final $\})$.

European Commission, 2020b. Communication from the Commission to the European Parliament and the Council. Shaping the conference on the future of Europe COM(2929) 27 final.

European Commission, 2021. A long-term Vision for the EU's Rural Areas-Towards stronger, connected, resilient and prosperous rural areas by 2040. COM(2021) 345 final. European Commission. Online: https:/ / ec.europa.eu/info/ sites/default/files/strategy/strategy_documents/documents/ltvra-c2021-345_en.pdf (accessed: 27 September 2021)

European Commission. Directorate General for Economic and Financial Affairs, 2020. The 2021 ageing report: Underlying assumptions and projection methodologies. Publications Office. Online: https://data.europa.eu/ doi/10.2765/733565 (accessed: 27 September 2021).

European Parliament and Council, 2021. Regulation (EU) 2021/1058 of the European Parliament and of the Council of 24 June 2021 on the European Regional Development Fund and on the Cohesion Fund. L 231/60. Official Journal of the European Union.

Fischer F., Miller G.J. (eds), 2017. Handbook of public policy analysis: Theory, politics, and methods. Routledge, New York DOI 10.4324/9781315093192.

Flick U. (ed.), 2014. The SAGE handbook of qualitative data analysis. SAGE Publications Ltd. London. ISBN 9781446208984

Gibbs G.R., 2014. Using Software in Qualitative Analysis. In Flick U., The SAGE Handbook of Qualitative Data Analysis SAGE Publications Ltd. London: 277-294. DOI 10.4135/9781446282243.n19.

Gløersen E., Drăgulin M., Hans S., Kaucic J., Schuh B., Keringer F., Celotti P., 2016. The impact of demographic change on European regions. European Union, Committee of the Regions. DOI 10.2863/26932.

Großmann K., Bontje M., Haase A., Mykhnenko V., 2013. Shrinking cities: Notes for the further research agenda. Cities 35: 221-225. DOI 10.1016/j.cities.2013.07.007.
Haase A., 2012. Shrinking areas front runners in innovative citizen participation. European Urban Knowledge Network.

Haase A., Bernt M., Großmann K., Mykhnenko V., Rink D., 2016. Varieties of shrinkage in European cities. European Urban and Regional Studies 23(1): 86-102. DOI $10.1177 / 0969776413481985$.

Haase A., Rink D., Grossmann K., Bernt M., Mykhnenko V., 2014. Conceptualizing urban shrinkage. Environment and Planning A: Economy and Space 46(7): 1519-1534. DOI $10.1068 / \mathrm{a} 46269$

Hecker S., Wicke N., Haklay M., Bonn A., 2019. How does policy conceptualise citizen science? A Qualitative Content Analysis of International Policy Documents. Citizen Science: Theory and Practice 4(1): 32. DOI 10.5334/cstp.230.

Hoekveld J.J., 2012. Time-space relations and the differences between shrinking regions. Built Environment 38(2): 179-195. DOI 10.2148/ benv.38.2.179.

Hollander J.B., Pallagst, K., Schwarz T., Popper F.J., 2009. Planning shrinking cities. Progress in Planning 72(4): 223-232.

Hospers G.J., 2013. Coping with shrinkage in Europe's cities and towns. Urban Design International 18(1): 78-89. DOI 10.1057/udi.2012.29.

Howland D., Becker M.L., Prelli L.J., 2006. Merging content analysis and the policy sciences: A system to discern policy-specific trends from news media reports. Policy Sciences 39(3): 205-231. DOI 10.1007/s11077-006-9016-5.

Krippendorff K., 2018. Content analysis: An introduction to its methodology (Fourth Edition). SAGE Publications, Inc; New York. ISBN: 978-1412983150.

Pallagst K., 2010. Viewpoint: The planning research agenda: Shrinking cities - a challenge for planning cultures. Town Planning Review 81(5): i-vi. DOI 10.3828/tpr.2010.22.

Pazos-Vidal S., 2021. Recognising demographically declining areas in EU Cohesion Policy: A case-study of multilevel coalition building. Regional Studies Association, Brighton. DOI 10.1080/13673882.2021.00001102.

Prior L., 2014. Content analysis. In Leavy P. (ed.), The Oxford Handbook of Qualitative Research. Oxford University Press, Oxford: 358-379. DOI 10.1093/oxford$\mathrm{hb} / 9780199811755.013 .008$.

Rink D., Haase A., Bernt M., 2009. Specification of working model. Work package 1 of Shrink Smart: Governance of shrinkage in a European context. Helmholtz Centre for Environmental Research, Leipzig.

Rodríguez-Pose A., 2018. The revenge of the places that don't matter (and what to do about it). Cambridge Journal of Regions, Economy and Society 11(1): 189-209. DOI 10.1093/ cjres/rsx024.

Rodríguez-Pose A., 2020. The rise of populism and the revenge of the places that don't matter. LSE Public Policy Review 1(1): 4. DOI 10.31389/lseppr.4.

Sarainsky N.G., 2015. Analyzing public discourse: Using media content analysis to understand the policy process. Columbia University 18(1): 26-41.

Schreier M. 2014. Qualitative content analysis. In Flick U., The SAGE Handbook of Qualitative Data Analysis SAGE Publications Ltd, London: 170-183. DOI 10.4135/9781446282243. n12.

SCiRN, 2008. COST action: Cities regrowing smaller. TU Dortmund.

Snelson C.L., 2016. Qualitative and mixed methods social media research: A review of the literature. International Journal of Qualitative Methods 15(1): 160940691562457. DOI $10.1177 / 1609406915624574$. 
Stryjakiewicz T., Jaroszewska E., 2016. The process of shrinkage as a challenge to urban governance. Quaestiones Geographicae 35(2): 27-37. DOI 10.1515/quageo-2016-0013.

The Economist, 2017. Why Europe needs more migrants Online: https://www.economist.com/graphic-detail/2017/07/12/why-europe-needs-more-migrants (accessed: 27 September 2021).

Venghaus S., Hake J.-F., 2018. Nexus thinking in current EU policies - The interdependencies among food, energy and water resources. Environmental Science \& Policy 90: 183-192. DOI 10.1016/j.envsci.2017.12.014.

Verma I., Taegen J., 2019. Access to services in rural areas from the point of view of older population-A case study in Finland. International Journal of Environmental Research and Public Health 16(23): 4854. DOI 10.3390/ ijerph16234854.
Wiechmann T., 2009. What are the problems of shrinking cities? Lessons learned from an international comparison. In Pallagst K. (ed.), The future of shrinking cities: Problems, patterns and strategies of urban transformation in a global context. University of California.

Wiechmann T., Wolff M., 2013. Urban shrinkage in a spatial perspective - Operationalization of Shrinking Cities in Europe 1990-2010. Conference Paper.

Wolff M., Fol S., Roth H., Cunningham-Sabot E., 2017. Shrinking cities: Measuring the phenomenon in France. Cybergeo DOI 10.4000/cybergeo.28033.

Wolff M., Wiechmann T., 2018. Urban growth and decline: Europe's shrinking cities in a comparative perspective 1990-2010. European Urban and Regional Studies 25(2): 122-139. DOI 10.1177/0969776417694680. 